\title{
Synthesis of Fibre Gratings
}

M. N. Zervas and R. Feced

Optoelectronics Research Centre

University of Southampton Southampton SO17 1BJ

UK

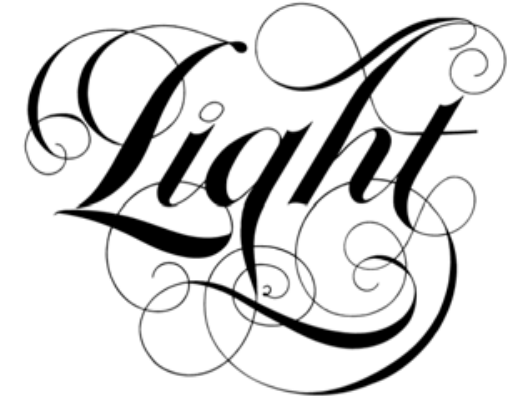




\title{
Acknowledgements
}

\author{
H-S Kim \\ M. Durkin \\ M. Ibsen \\ V. Finazzi
}




\section{Outline}

- Introduction (Analysis / Synthesis)

- Grating Synthesis (Design) Methods

- Fourier-Transform Methods

- Integral Methods

- Differential Methods

- Layer-Peeling IS Method

- Grating Designs

- Square Dispersionless Filters

- Dispersion Compensators

- 2nd \& 3rd order

- Conclusions 


\section{Grating Analysis - Synthesis}

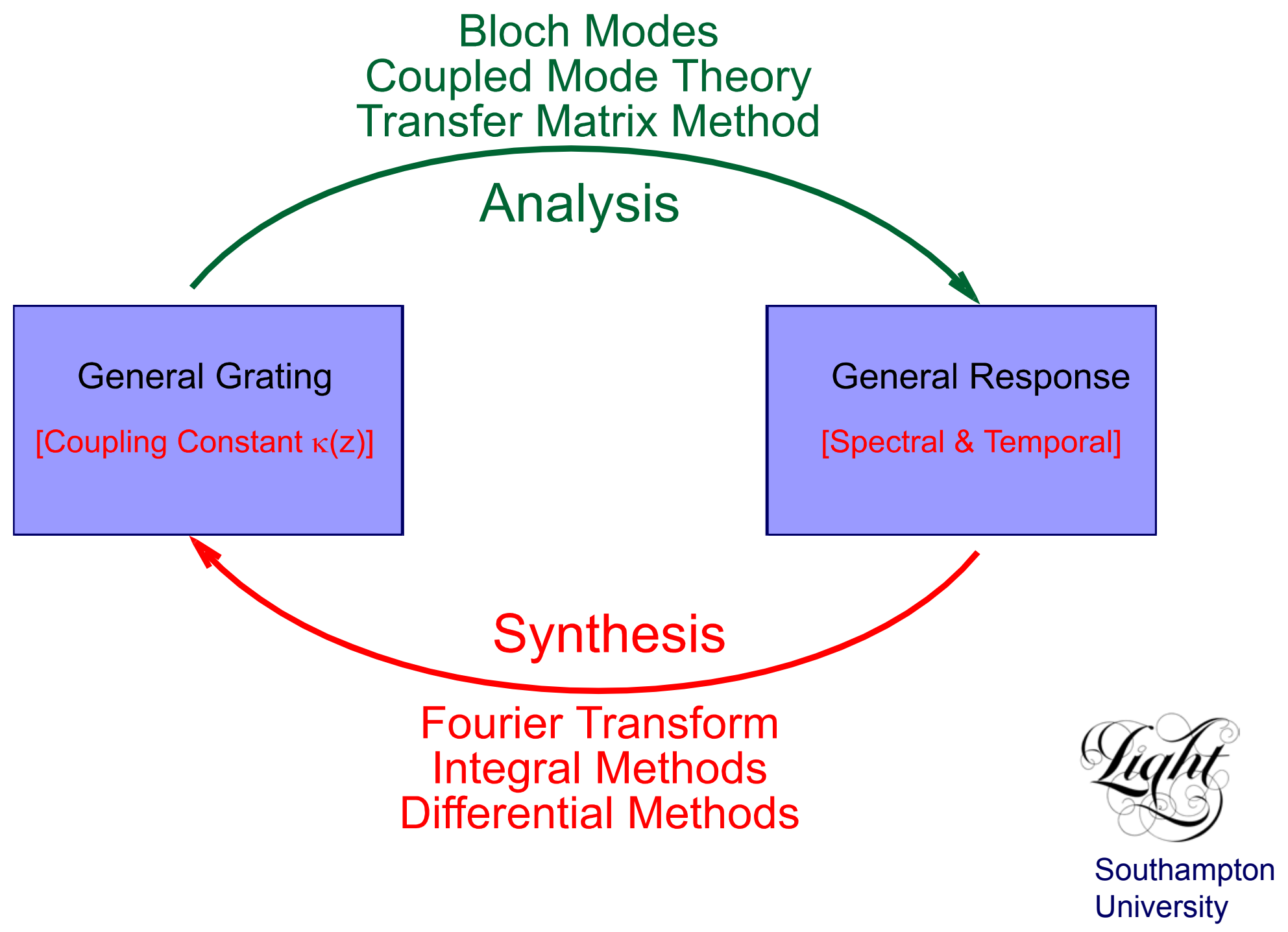




\section{Grating Reflection}

Multiple Distributed Scattering

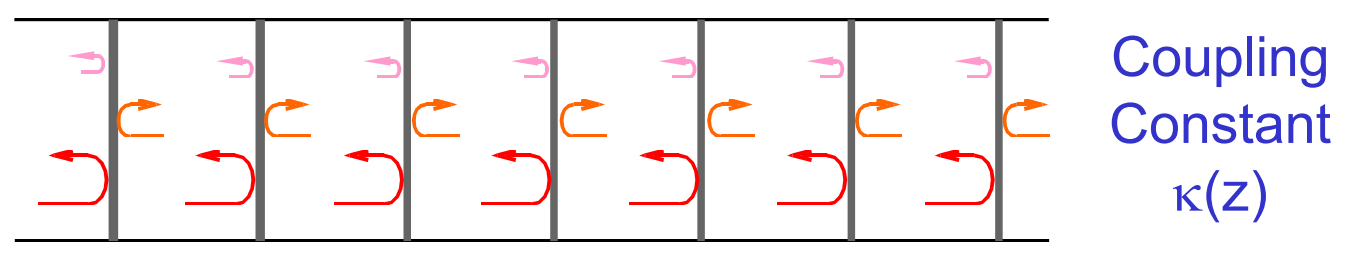

equivalent to

Two oppositetravelling waves

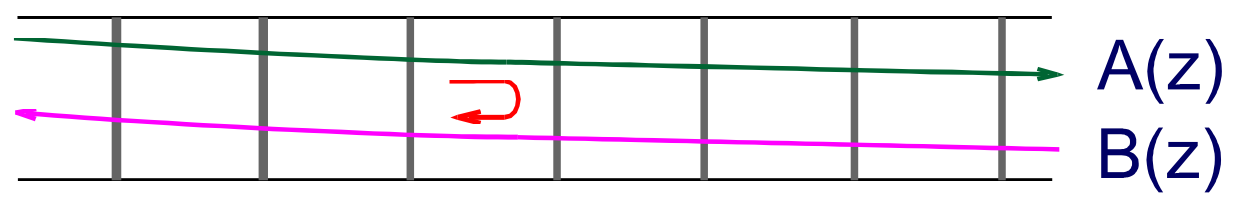

Local Reflection Coefficient $\quad r(z)=\frac{B(z)}{A(z)}$

$$
\text { Ricatti Equation } \quad \frac{d r}{d z}=-i 2 \beta r+\kappa-\kappa^{*} r^{2}
$$




\section{General Grating Reflection Coefficient}

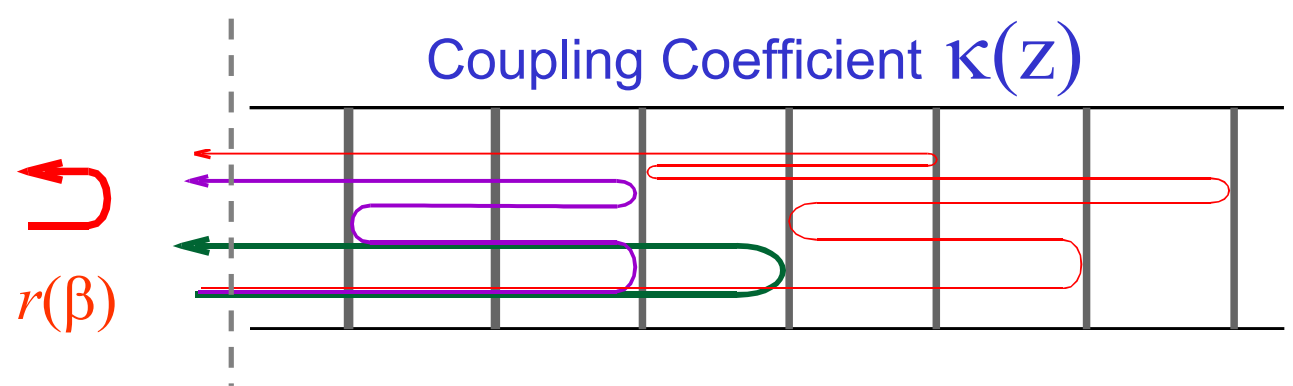

$$
\begin{aligned}
r(\beta)= & -\int_{z_{1}=0}^{L} d z_{1} \kappa\left(z_{1}\right) e^{i \beta 2 z_{1}} \\
& +\int_{z_{1}=0}^{L} d z_{1} \int_{z_{2}=0}^{z_{1}} d z_{2} \int_{z_{3}=z_{2}}^{L} d z_{3} \kappa\left(\mathrm{z}_{1}\right) \kappa^{*}\left(\mathrm{z}_{2}\right) \kappa\left(\mathrm{z}_{3}\right) e^{i \beta 2\left(z_{1}-z_{2}+z_{3}\right)} \\
& -\int_{z_{1}=0}^{L} d z_{1} \int_{z_{2}=0}^{z_{1}} d z_{2} \int_{z_{3}=z_{2}}^{L} d z_{3} \int_{z_{4}=0}^{z_{3}} d z_{4} \int_{z_{5}=z_{4}}^{L} d z_{5} \kappa\left(\mathrm{z}_{1}\right) \kappa^{*}\left(\mathrm{z}_{2}\right) \kappa\left(\mathrm{z}_{3}\right) \kappa^{*}\left(\mathrm{z}_{4}\right) \kappa\left(\mathrm{z}_{5}\right) e^{i \beta 2\left(z_{1}-z_{2}+z_{3}-z_{4}+z_{5}\right)} \\
& +\ldots \text { (Higher Order Reflections) }
\end{aligned}
$$




\section{Main Grating Design Methods}

- Fourier Method

- Integral Methods

- Differential Methods

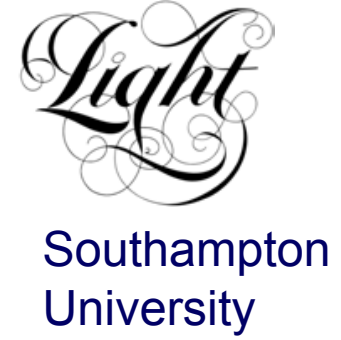




\section{Grating Design - Fourier Method}

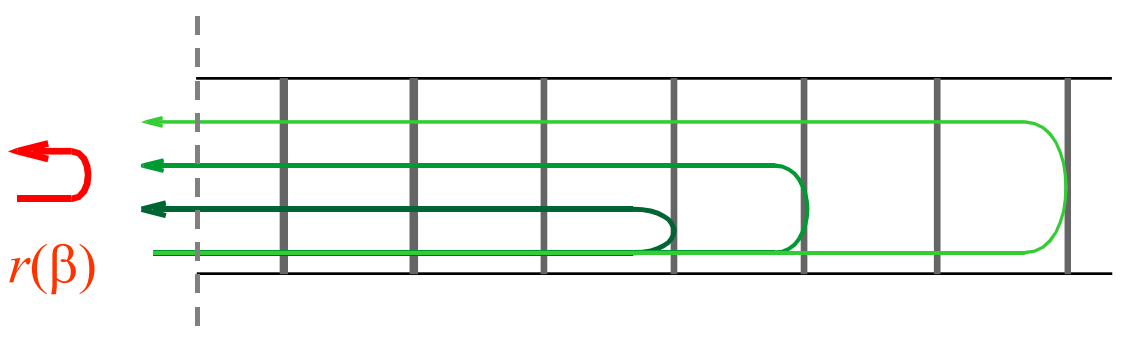

For weak gratings $(r<<1)$

$$
r(\beta)=-\int_{z_{1}=0}^{L} d z_{1} \kappa\left(z_{1}\right) e^{i \beta 2 z_{1}}
$$

$$
\kappa(z)=-\frac{1}{\pi} \int_{-\infty}^{+\infty} r(\beta) e^{-i 2 \beta z} d \beta
$$

$$
r(\beta) \stackrel{\mathrm{FT}}{\longleftarrow} \kappa(\mathrm{z})
$$

- Accurate only for low reflectivities

- Limited but quite useful 


\section{Grating Design - Fourier Method}

Coupling Constant

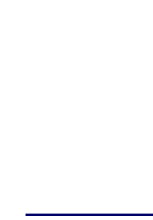

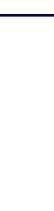
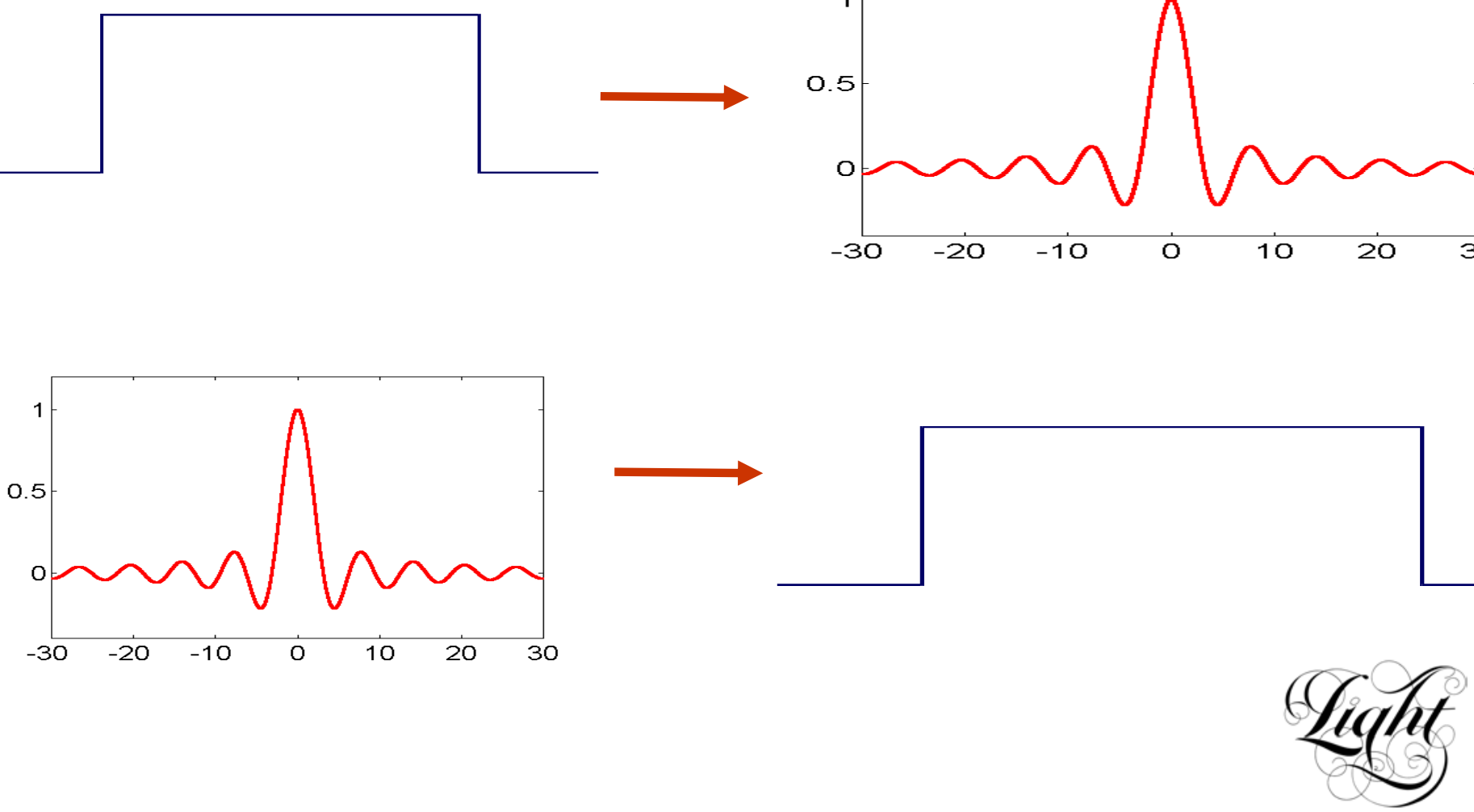

Southampton University 


\section{Integral IS Methods \\ [Gelfand-Levitan \& Marchenko (GLM) Method]}

- Invert Grating Response in Fourier Domain

- Coupling Constant in terms of Generalised FT Integral

- Exact Method - Multiple reflections accounted for

- Solution of Integral Equations

- Analytic solution exists when $r(\beta)$ is rational function

- Solution usually involves (NxN) matrices

- Iterative solutions have been proposed

- High Algorithmic Complexity: $\mathrm{O}\left(\mathrm{N}^{3}\right)$ 


\title{
Differential IS Methods
}

\author{
[Layer-Peeling Methods]
}

- Invert Grating Response in Time Domain

- Rely on Causality

- Exact Method - Multiple reflections accounted for

- Solution of Difference Equations

- Layer-by-layer medium identification

- Replicate Scattering Physical Process

- Low Algorithmic Complexity: $\mathrm{O}\left(\mathrm{N}^{2}\right)$ 


\section{Layer-Peeling Grating Design Method}

- General Description

- Space-Time Diagrams

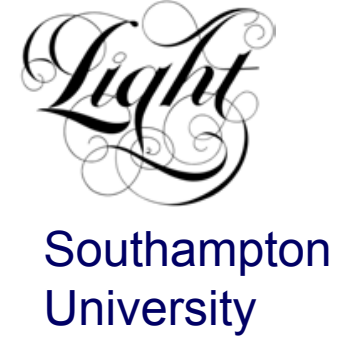




\section{Layer-Peeling IS Algorithm}

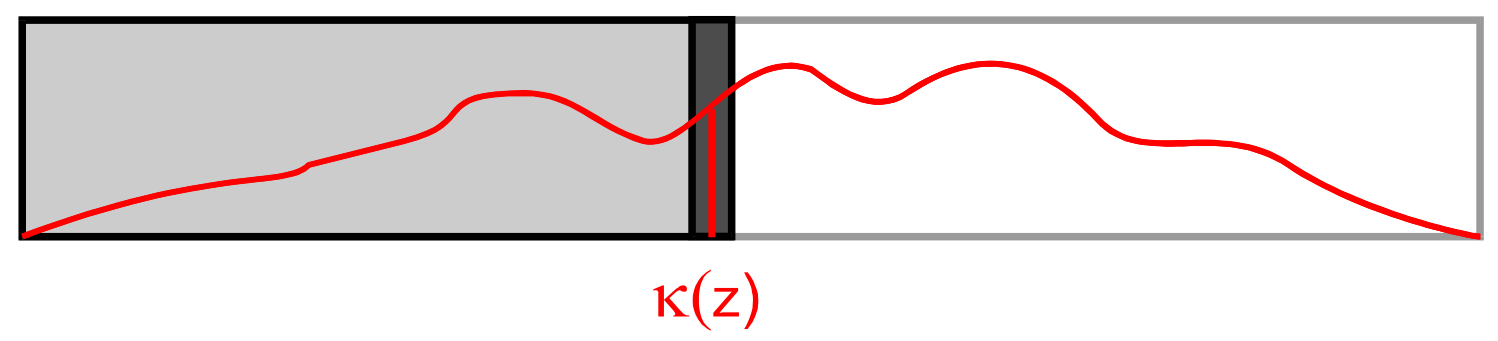

Step: 1) Impulse Response $\left[h_{R}(\tau)\right]$ of Desired Medium $\quad[\rightarrow$ FT of $r(\beta)]$

$\longrightarrow 2$ ) Impulse Response $\left[h_{T}(\tau)\right]$ of Identified Medium $\left[\rightarrow F T\right.$ of $\left.r_{T}(\beta)\right]$

$$
\text { 3) } \quad \kappa(\tau / 2)=-2\left[h_{R}(\tau)-h_{T}(\tau)\right]
$$




\section{Multiple Scattering Reflection}

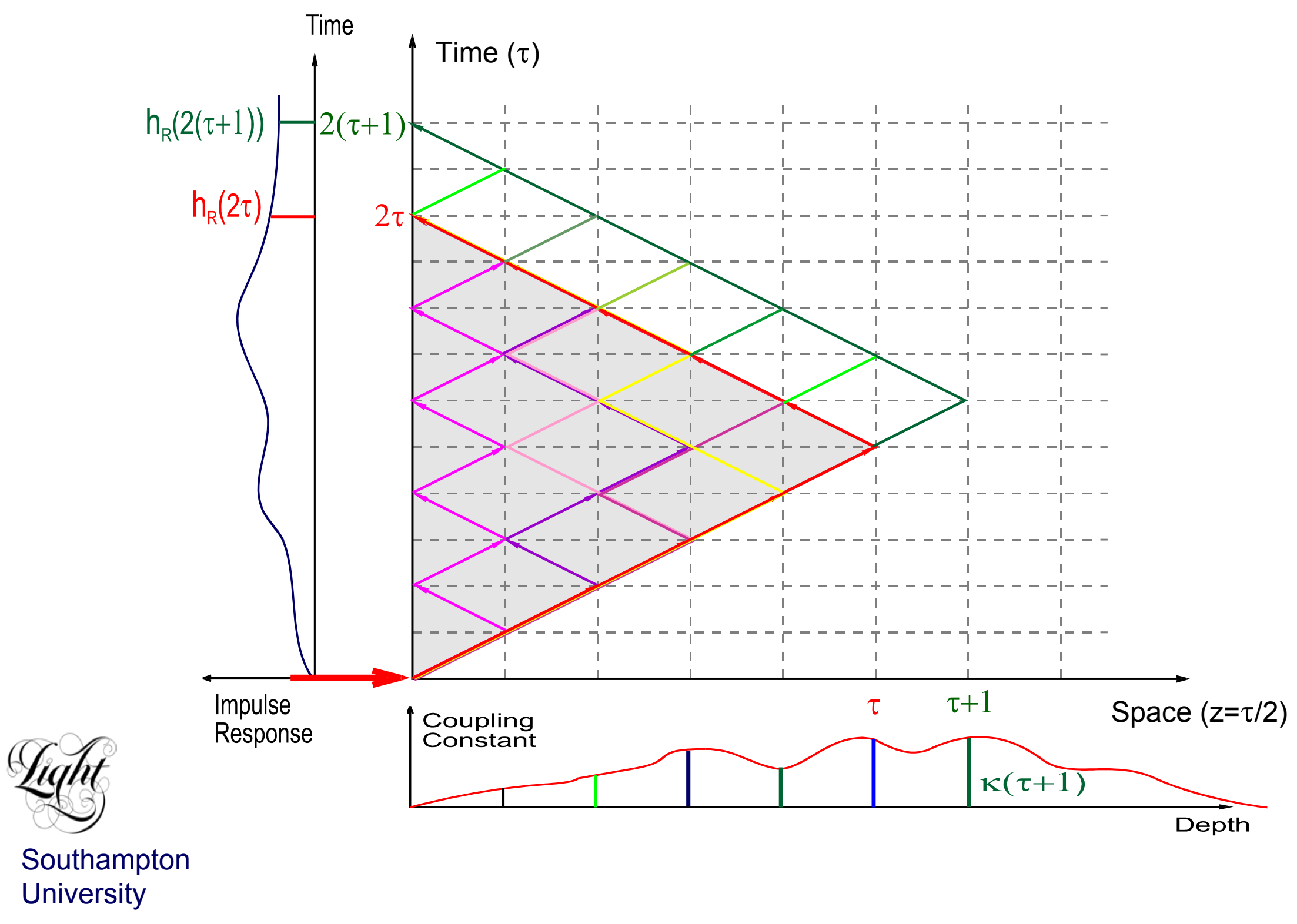




\section{Layer-Peeling Inverse Scattering Method}

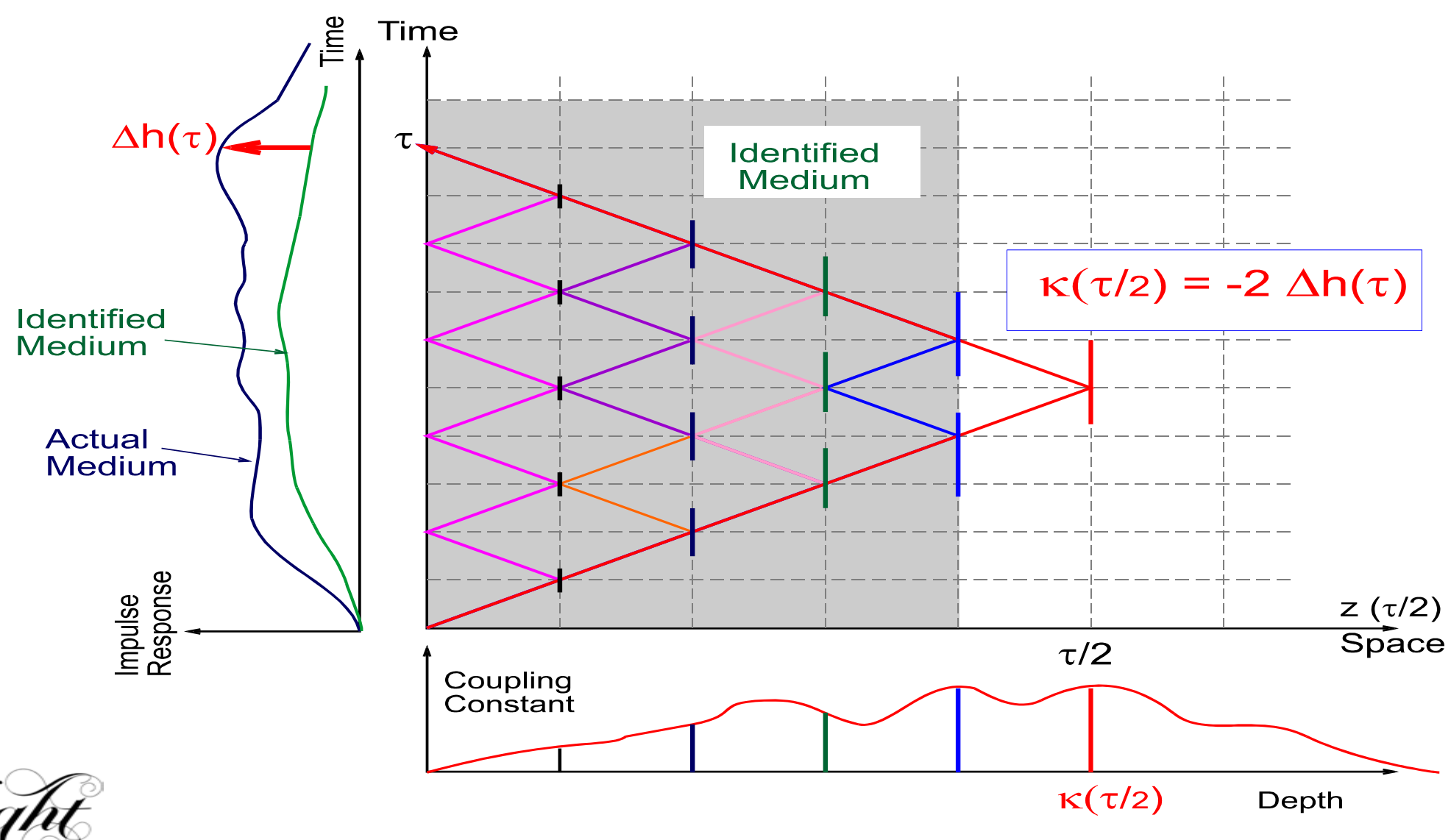

Southampton

University 


\section{Layer-Peeling Method Grating-Design Examples}

- Uniform Grating Reconstruction

- Square Dispersionless Filters

- 2nd-order Dispersion Compensators

- 3rd-order Dispersion Compensators 


\section{Uniform Grating Reconstruction}

Layer-Peeling -vs- Iterative GLM

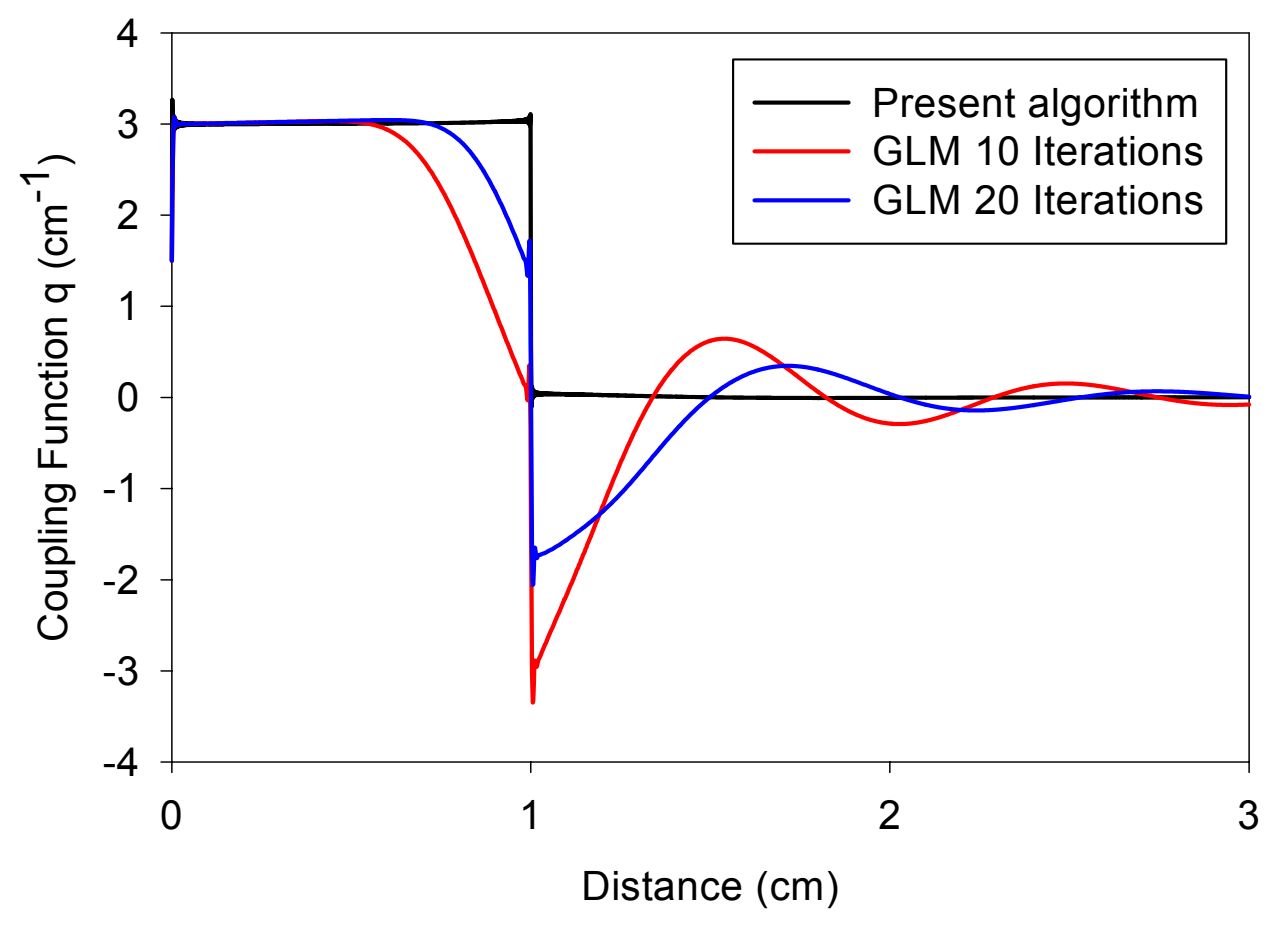




\section{Square Dispersionless Filter Design}
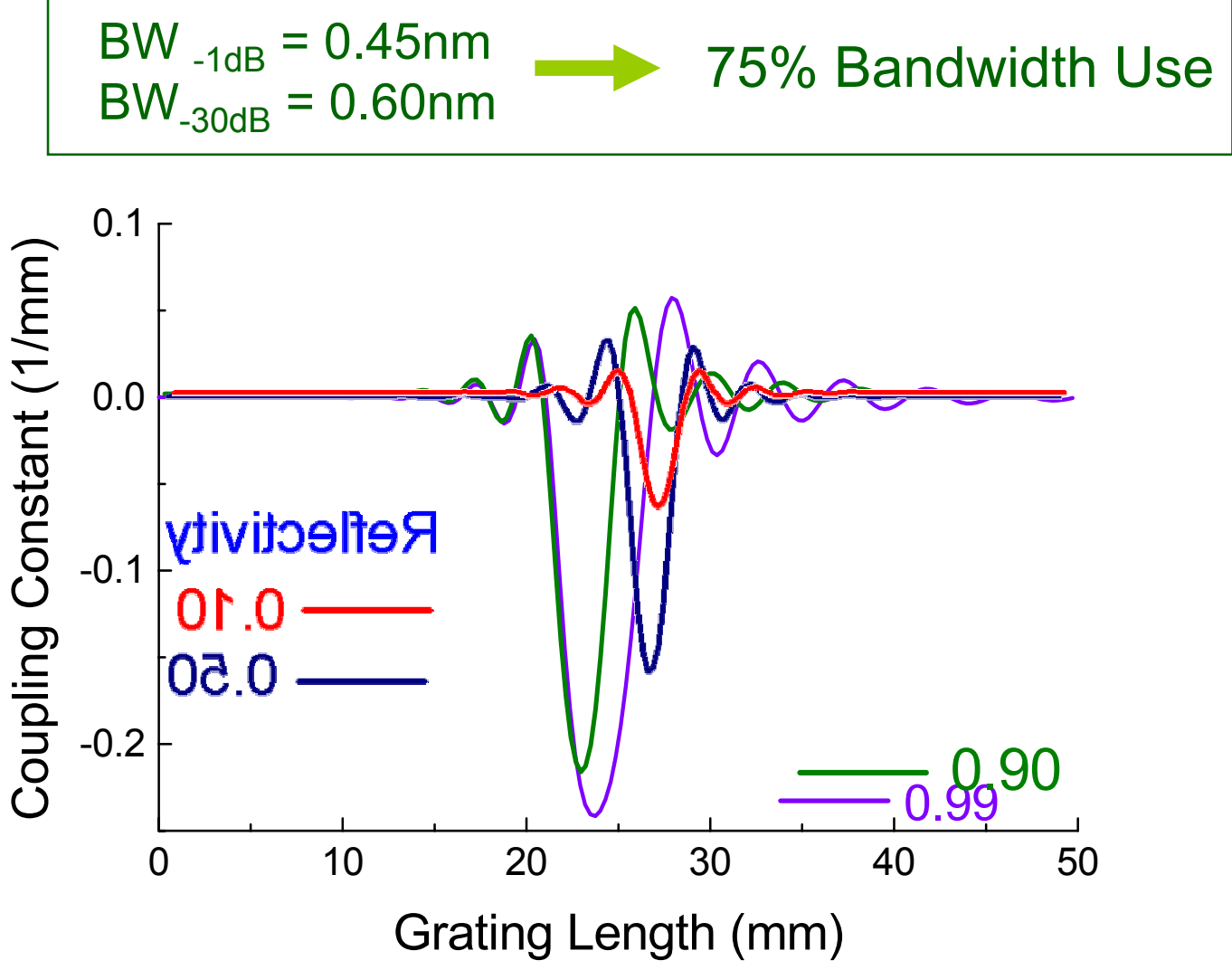


\section{Square Dispersionless Filters}

\section{Bandwidth Utilisation $=\mathrm{BW}_{-1} / \mathrm{BW}_{-30}$}

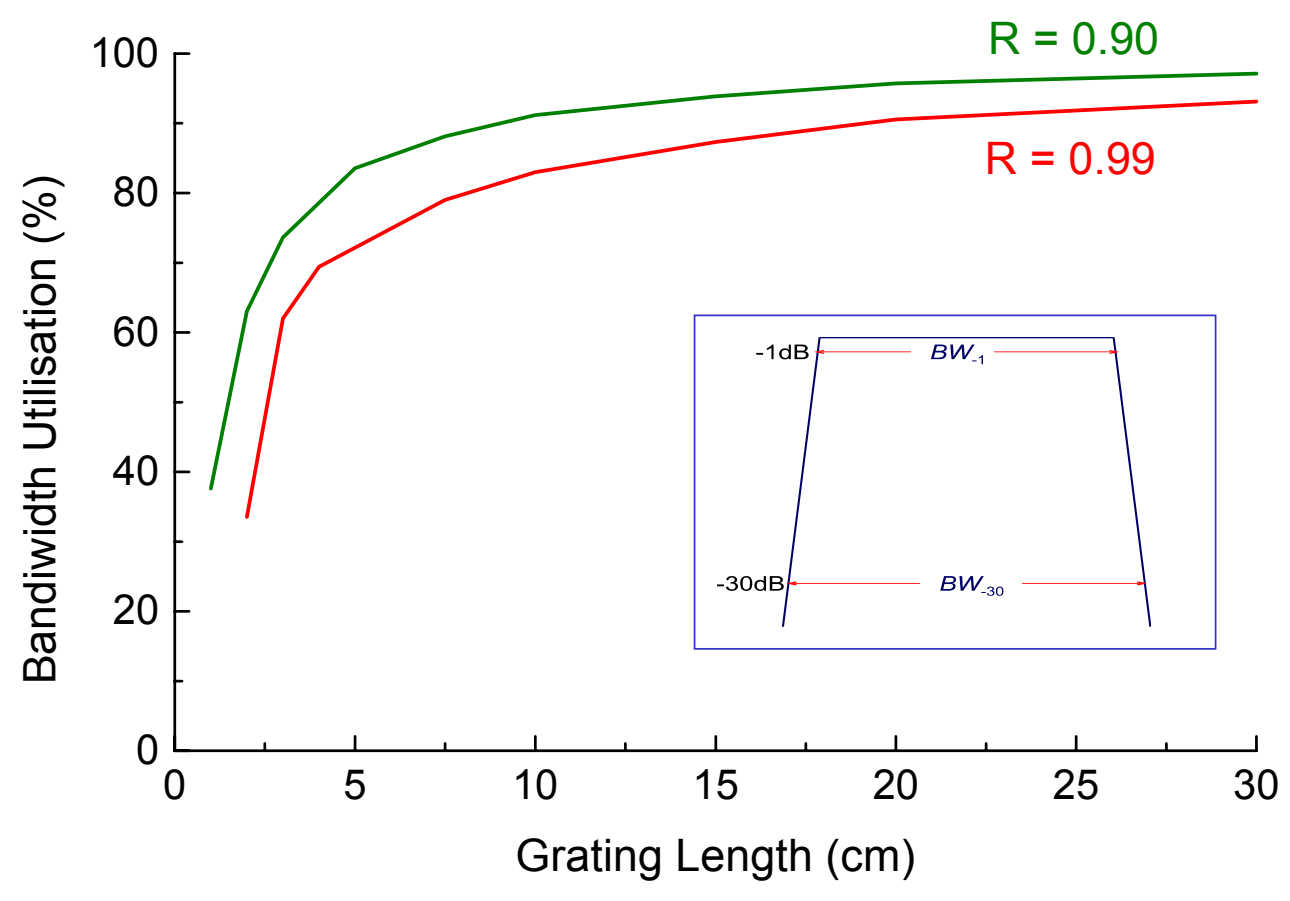




\section{Linear Dispersion Compensator Grating Design}

Layer-Peeling

IS Design
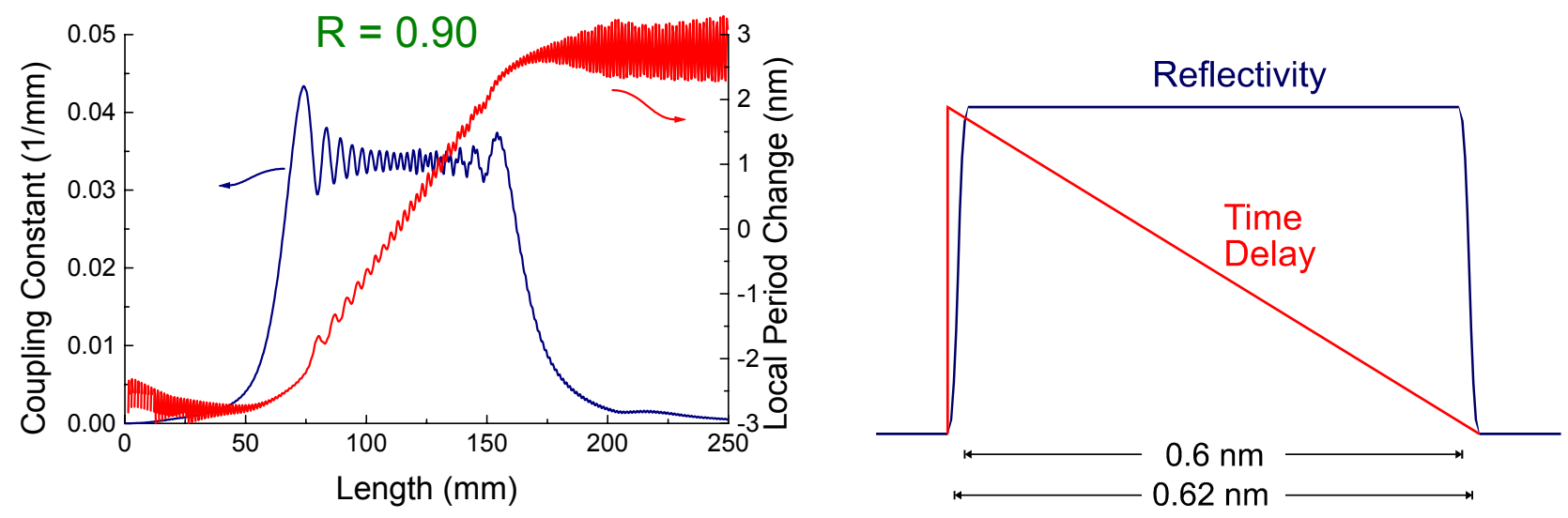

Conventional

Design

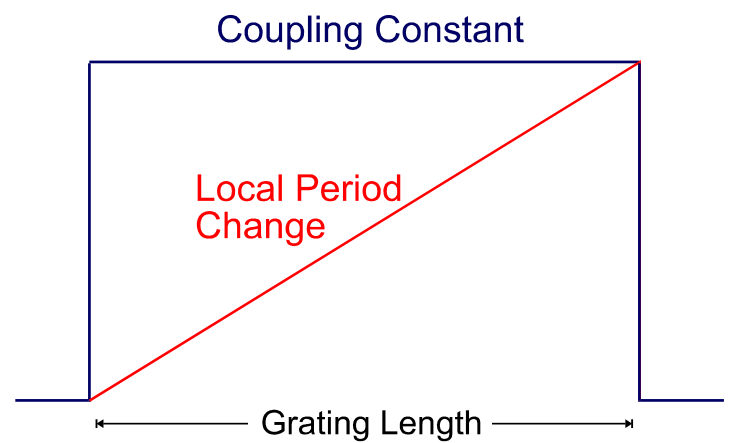

\section{cight}

Grating Length

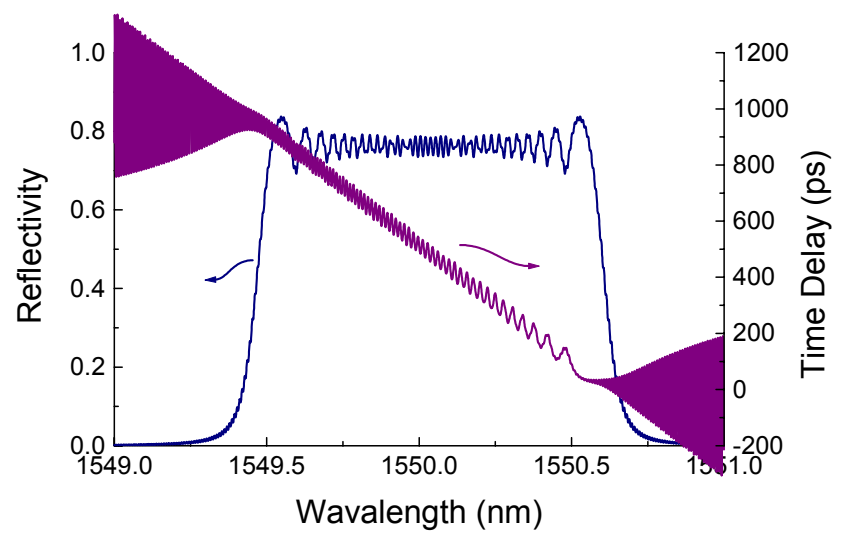

Southampton University 


\section{2nd order Grating Dispersion Compensator Design}
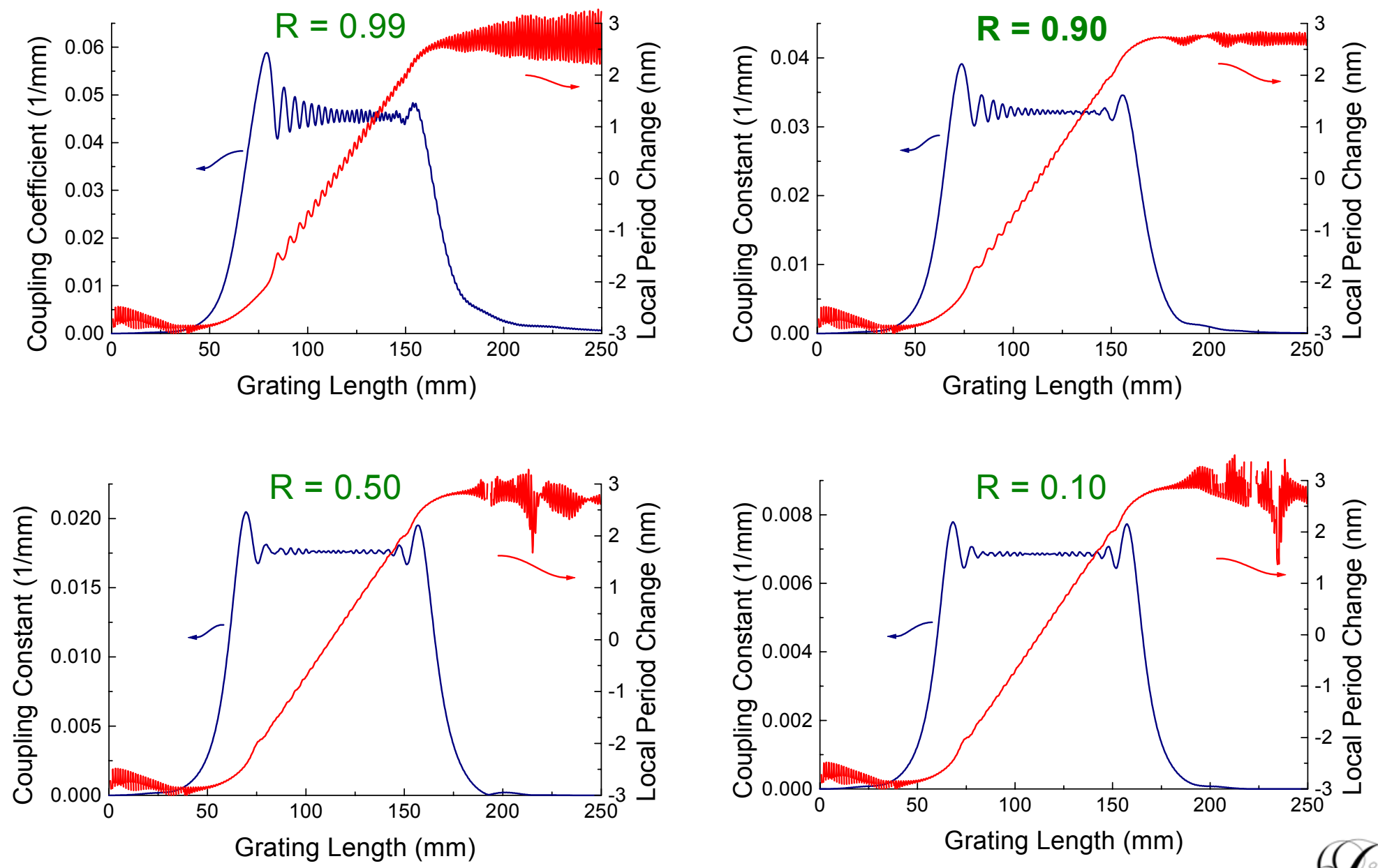

trifht

Southampton University 


\section{2nd order Grating Dispersion Compensator Design}
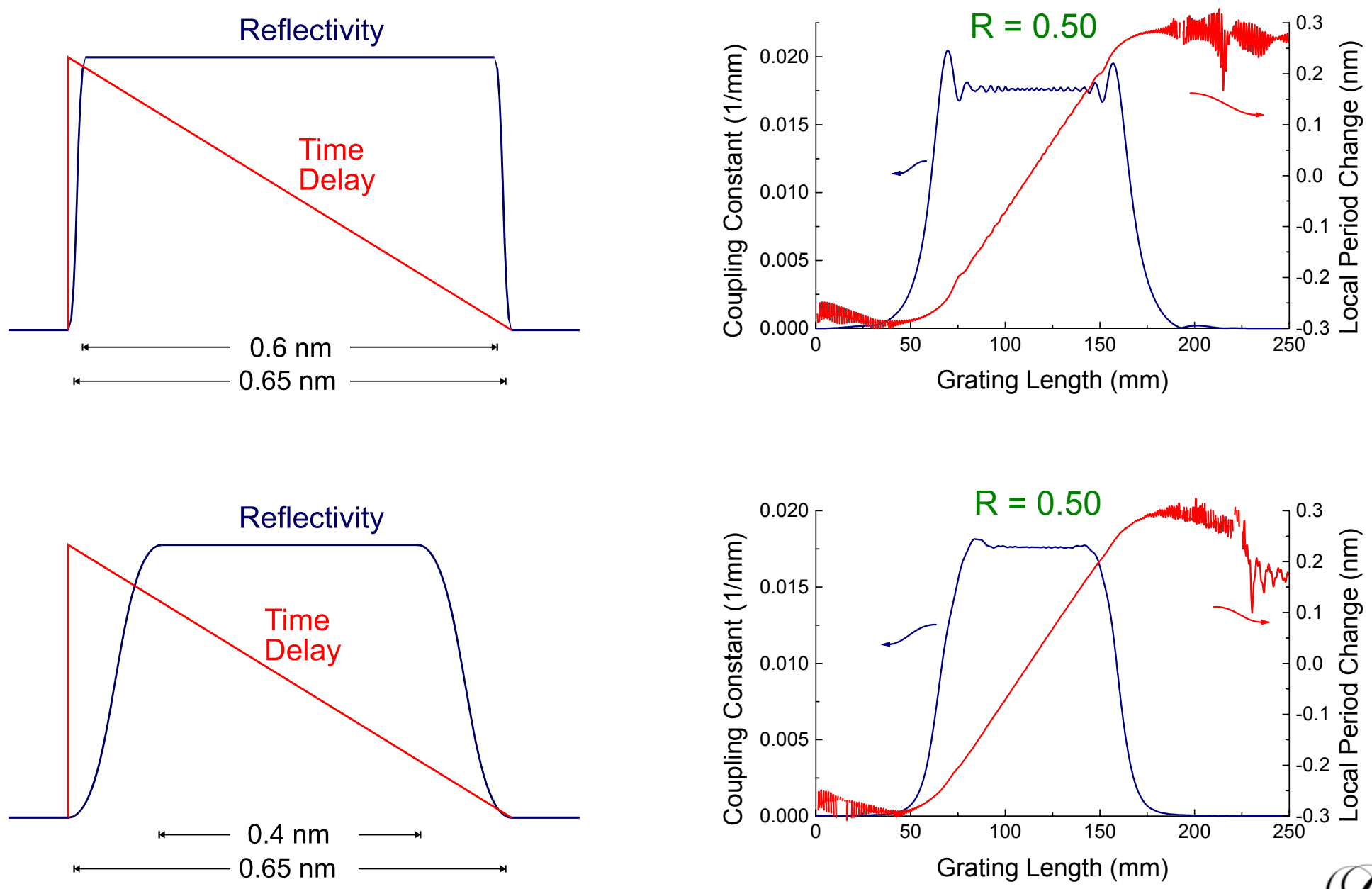

Southampton University 


\section{3rd Order Dispersion Compensators}
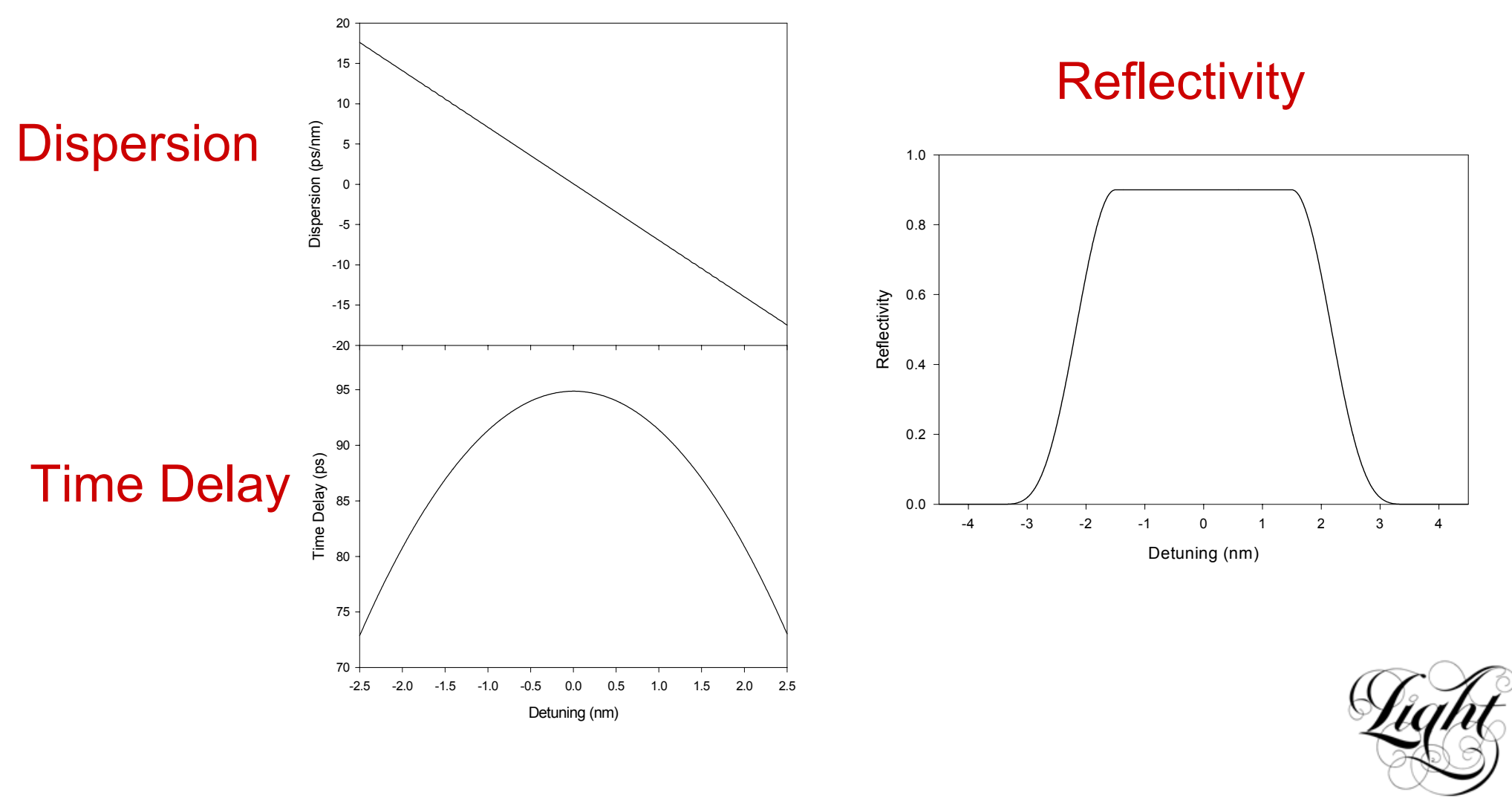

Southampton University 


\section{3rd Order Dispersion Compensator Design}

- Real Coupling Coefficient

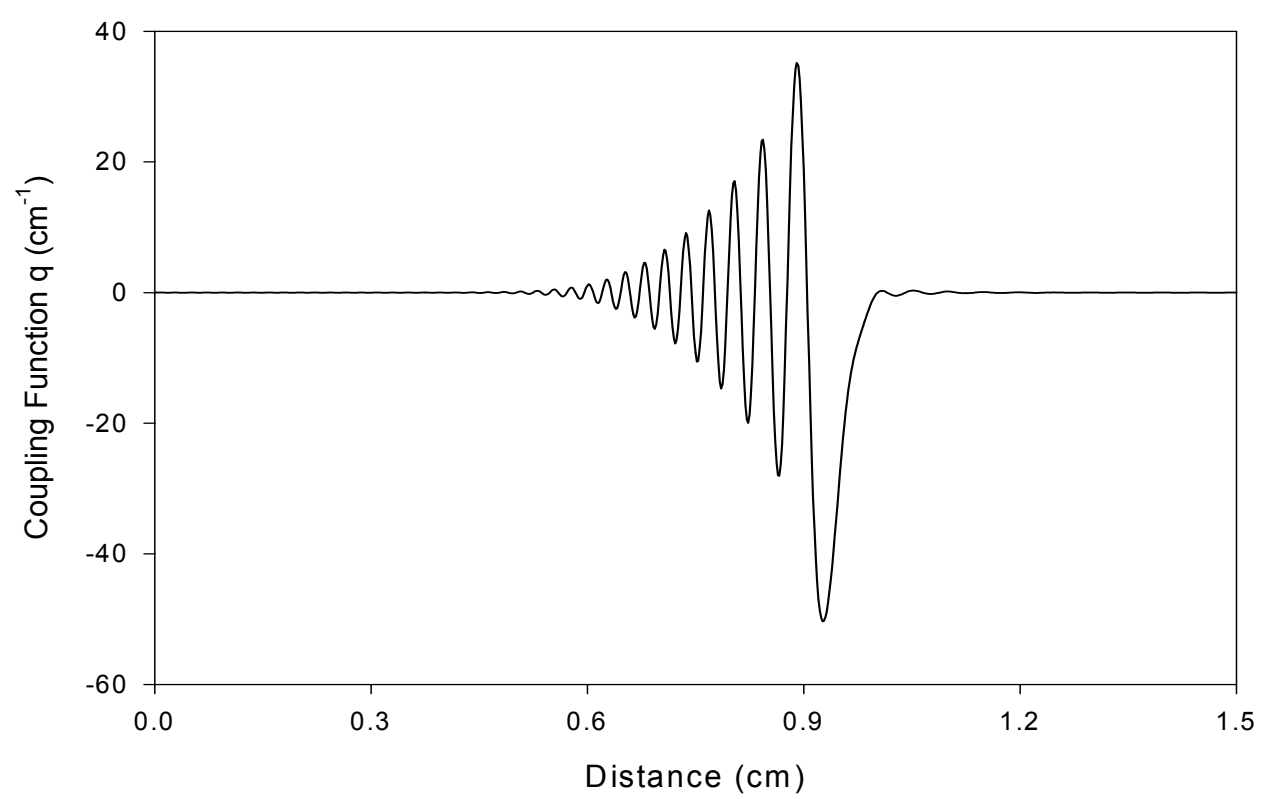

- Constant Period 


\section{3rd order DC - Physical Picture}

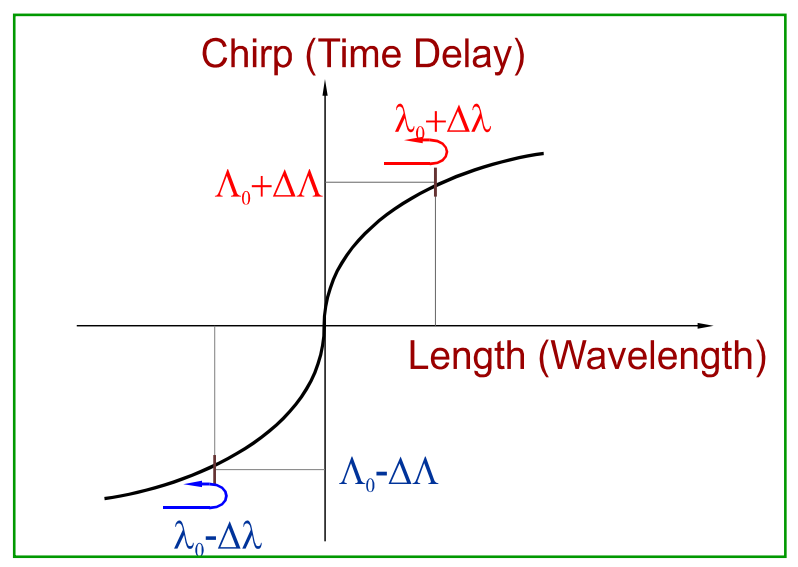

Chirped Compensator (with Antisymmetric Chirp)

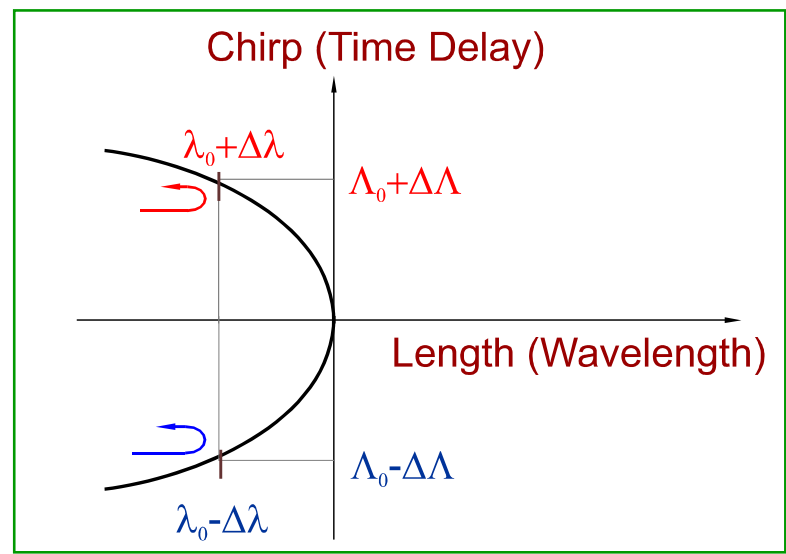

Folded Chirped Compensator

Unchirped

Locally Varying Moire Grating

Southampton University 


\section{Conclusions}

- Main grating-design techniques have been reviewed

- Differential Layer-Peeling Method presented extensively

Extremely Powerful

Replicates physical scattering process

Fast \& Accurate method

Gives exact solutions to exact scattering problems

- Provides Novel Exciting Grating Designs

- Enhances Grating Design Intuition

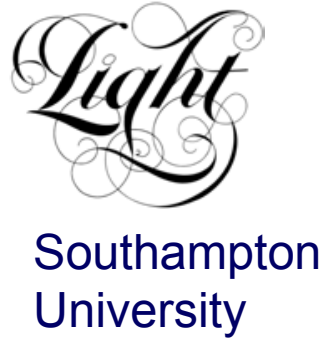

